\title{
DESENVOLVIMENTO DE UM MÉTODO ANALÍTICO RÁPIDO E EFICIENTE PARA A DETERMINAÇÃO DE CORTICOSTERÓIDES PLASMÁTICOS POR INJEÇÃO DIRETA EM COLUNA CROMATOGRÁFICA ISRP-C ${ }_{18}$ POR CLAE.
}

\author{
E.A. Toledo-Pinto ${ }^{1}$, M.L. Menezes ${ }^{1}$, O.C.M. Pereira ${ }^{2}$ \\ ${ }^{1}$ Departamento de Química, Faculdade de Ciências, Universidade Estadual Paulista, Av. Edmundo Carrijo Coube $n^{\circ}$ 14-01, \\ CEP 17033-360, Bauru, São Paulo, Brasil. \\ ${ }^{2}$ Departamento de Farmacologia, Instituto de Biociências, Universidade Estadual Paulista, Distrito de Rubião Jr., s/n ${ }^{o}$, \\ CEP 18618-000, Botucatu, São Paulo, Brasil. \\ elianetol@hotmail.com
}

\begin{abstract}
Resumo: O presente trabalho teve como objetivo o desenvolvimento de um método analítico simples, rápido e eficiente para a determinação de corticosteróides (cortisona, corticosterona, acetato de hidrocortisona e acetato de dexametasona) em amostras sangüíneas de ratos empregando um sistema isocrático de cromatografia líquida de alta eficiência (CLAE) com detecção ultravioleta. O método envolveu a injeção direta da amostra sangüínea em uma coluna cromatográfica com superfície interna de fase reversa (ISRP-C ${ }_{18}$ ), empregando a fase móvel composta por tampão fosfato $\mathrm{pH} 4,0$ : acetonitrila $(65: 35 \mathrm{v} / \mathrm{v})$. A detecção dos analitos foi obtida, através de um detector de ultravioleta de comprimento de onda variável (Varian Modelo 2550) ajustado em $240 \mathrm{~nm}$ e um integrador SP 4400 Chromaject (Varian Associates, Inc, Sunnyvale, CA, USA). A extração do analito resultou em valores de recuperação entre $90 \%$ e $108 \%$, e coeficiente de variação entre $1,1 \%$ a $2,5 \%$. Os limites de detecção e quantificação do método foram de $0,02 \mathrm{e}$ $0,04 \mu \mathrm{g} \mathrm{mL} \mathrm{m}^{-1}$, respectivamente. Assim, o método analítico proposto possibilitou a injeção direta (on-line) da amostra sem tratamento prévio apresentando também várias vantagens, tais como: rapidez, exatidão, precisão e especificidade.
\end{abstract}

Palavras-chave: corticosteróides, amostra biológica, coluna ISRP-C ${ }_{18}, \mathrm{CLAE}$.

\section{INTRODUÇÃO}

\section{Corticosteróides}

Os corticosteróides também chamados de adrenocorticosteróides são hormônios de natureza esteroídica produzidos na porção cortical das glândulas adrenais, que estão localizados exata- mente no pólo superior de ambos os rins [1]. Estes hormônios são sintetizados a partir do colesterol e constituem três famílias: os glicocorticóides, os mineralocorticóides e os andrógenos adrenais. Os glicocorticóides atuam primariamente no metabolismo das proteínas, carboidratos e lipídios, e os principais hormônios endógenos são o cortisol e a corticosterona, como exemplos de sintéticos: cortisona, prednisona, metilprednisona, dexametasona, entre outros [2]. Os mineralocorticóides atuam 
quase exclusivamente em tecidos de troca iônica, reabsorvendo sódio e fluídos, excretando potássio e hidrogênio (equilíbrio eletrolítico), o principal hormônio endógeno é a aldosterona, entre os sintéticos têm-se: $9 \alpha$-fluorocortisol e cortisona [3]. Os androgênios ou estrogênios atuam sobre as características sexuais secundárias em seus órgãos específicos.

\section{Considerações sobre análise de corticosteróides}

Radioimunoensaio e ensaios com ligantes protéicos competitivos são freqüentemente usados como métodos de escolha para dosagem de cortisol em humanos. Embora estes métodos sejam muito sensíveis, não possuem alta especificidade. A necessidade de desenvolver uma metodologia mais rápida, precisa e específica para analisar corticosteróides em amostras biológicas conduziu muitos pesquisadores a utilizar a cromatografia ou cromatografia líquida de alta eficiência.

Usualmente para analisar corticosteróides, é necessário um pré-tratamento das amostras de urina incluindo hidrólises enzimáticas, e extração líquido-líquido (LLE) ou extração de fase sólida (SPE) $[1,4,5,6,7]$. Já a determinação de corticosteróides em plasma por cromatografia líquida consiste em extração das proteínas e purificação dos analitos antes de injetá-los no sistema.

Separação de hormônios esteróides por CLAE com detecção ultravioleta e fluorescência têm sido amplamente utilizados. Estes métodos permitem uma determinação bem específica dos corticosteróides tanto na urina como no plasma, providenciando um bom método para as análises [6].

Diferentes métodos de CLAE para separação de compostos e misturas complexas de corticosteróides utilizando convencionalmente fases móveis têm sido recentemente desenvolvidos e aplicados para amostras de urina, plasma e formulações farmacêuticas $[4,5,6,7,8,9,10,11]$. Em todos os métodos abordados constatou-se que houve a necessidade de um pré-tratamento da amostra empregando a técnica de extração em fase sólida ou extração por solventes. Embora as técnicas off-line sejam mais seletivas e modernas, alguns problemas ainda persistem como, por exemplo: o tempo gasto no preparo das amostras, manuseio laborioso nesta etapa e os materiais empregados no desenvolvimento de um método, ou são descartáveis, ou têm tempo de vida curto. Assim, o uso de procedimentos on-line vem se destacando em decorrência de suas vantagens: eliminação dos erros humanos de manipulação, diminuição do tempo de assistência do analista durante a análise, minimização de perdas de analito, melhora na precisão e exatidão do método e segurança oferecida por esta técnica ao analista, uma vez que o manuseio de amostras perigosas ou infecciosas é reduzido $[12,13]$.

Os métodos supracitados não foram validados corretamente, com exceção dos trabalhos de Marwah e cols. (2001), Cirimele e cols. (2000) e Hay e Morméde (1997) [4, 6, 8], fato importantíssimo em análises quantitativas e qualitativas por cromatografia e por outros métodos analíticos.

O objetivo do presente trabalho foi desenvolver um método analítico simples, rápido e eficiente para a determinação de corticosteróides em amostras sangüíneas de ratos empregando coluna cromatográfica ISRP- $\mathrm{C}_{18}$ e análise por CLAE.

\section{MATERIAIS E MÉTODOS}

\section{Reagentes}

Os padrões, cortisona, corticosterona, acetato de dexametasona, acetato de hidrocortisona e albumina de soro humano foram adquiridos junto a Sigma - Aldrich Chemical Company (USA). O fosfato monossódico 1-hidrato, acetonitrila, metanol (grau CLAE) e o dissulfito de sódio foram obtidos junto a Merck, Merck KgaA (Darmsradt, Germany). A acetonitrila foi obtida da Carlo Erba (Milan, Italy). A água deionizada foi obtida a partir de um sistema de purificação Milli-Q, 
adquirido da Millipore, (Millipore, Bedford, MA, USA).

As soluções-padrão foram preparadas efetuando-se a solubilização de $0,005 \mathrm{~g}$ de cada corticosteróide (cortisona, corticosterona, acetato de dexametasona e acetato de hidrocortisona) em $10 \mathrm{~mL}$ de metanol. A partir desta solução estoque ( $500 \mu \mathrm{g} \mathrm{mL}^{-1}$ ) foram realizadas diluições obtendose concentrações de corticosteróides para a construção de curvas analíticas e determinação do limite de deteç̧ão.

\section{Animais}

Foram utilizados ratos albinos adultos machos, da variedade Wistar, com aproximadamente 90 dias de idade, pesando cerca de 300 gramas, fornecidos pelo Biotério Central da UNESP localizado no Campus de Botucatu. Os experimentos foram aprovados pelo Comitê de Ética em Experimentação Animal do Instituto de Biociências da Unesp, Botucatu.

Os animais permaneceram no Biotério do Departamento de Farmacologia do Instituto de Biociências da Unesp, por um período mínimo de 10 dias, sob condições controladas de alimentação, temperatura, ruído e ciclo claro-escuro para aclimatação. Estes animais foram mantidos em gaiolas coletivas, contendo cinco animais por gaiola, com livre acesso à água e alimento.

\section{Instrumentação}

Os experimentos cromatográficos foram realizados sob condições isocráticas em um sistema de cromatografia líquida de alta eficiência (Varian Modelo 2510), equipado com uma bomba recíproca, um detector de ultravioleta de comprimento de onda variável (Varian Modelo 2550) ajustado em $240 \mathrm{~nm}$ e um integrador SP 4400 Chromaject (Varian Associates, Inc, Sunnyvale, CA, USA). As amostras e as soluções-padrão foram injetadas em uma coluna ISRP-C ${ }_{18}(100 \mathrm{~mm} \times$ x 4,6 mm DI), com uma válvula manual de injeção, (Rheodyne 7125 ,
Cotati, Ca, USA), ligada a uma alça de injeção de $100 \mu \mathrm{L}$.

\section{Condições cromatográficas}

A separação de corticosteróides foi realizada à temperatura de $25^{\circ} \mathrm{C}$ constante, com vazão da fase móvel ajustado em $0,85 \mathrm{~mL} \mathrm{~min}^{-1}$. A fase móvel ideal encontrada foi composta por uma mistura de tampão fosfato $\mathrm{pH} 4,0$ e acetonitrila (65: $35 \mathrm{v} / \mathrm{v})$. O tempo de estabilização do sistema de CLAE foi de aproximadamente 15 minutos antes do início dos procedimentos experimentais.

\section{Métodos}

\section{Imobilização da albumina de soro humano in situ sobre a superfície da fase estacionária $\mathrm{Si}-\mathrm{C}_{18}$}

O método de imobilização das proteínas in situ empregado seguiu o protocolo de Menezes e cols. (1999) e Pompon e cols. (1992) [14, 15] partindo de uma coluna cromatográfica $\mathrm{C}_{18}(100 \mathrm{~mm}$ x 4,6 mm DI), Phenomenex - Luna $5 \mu \mathrm{m}$, avaliada comercialmente, adquirida junto a Labtron Comércio e Representações Ltda.

A determinação dos teores de proteínas imobilizadas sobre a superfície das sílicas foi determinada pela injeção de plasma diluído (1:100) fortificado com corticosteróides $[13,16,17,18]$ nas concentrações de $0,15,0,30,0,60,1,20$ e 1,6 $\mu \mathrm{g} \mathrm{mL} \mathrm{m}^{-1}$.

\section{Avaliação da composição da fase móvel}

Para a seleção da melhor fase móvel foram otimizadas as condições cromatográficas de três fases na separação dos corticosteróides, tampão fosfato $\mathrm{pH} 4,0$ : acetonitrila nas proporções de 50 : 50, 60: 40 e 65: $35(\mathrm{v} / \mathrm{v})$ com uma alça de 100 $\mu \mathrm{L}$. Para a escolha adequada da fase móvel foram 
realizados os cálculos para determinação dos parâmetros cromatográficos.

\section{Avaliação da vazão}

Para a seleção da vazão adequada para a determinação de corticosteróides foram avaliados os seguintes intervalos $0,70,0,75,0,80,0,85$, $0,90,0,95$ e $1,0 \mathrm{~mL}$ min.

\section{Avaliação da coluna cromatográfica após a imo- bilização das proteínas (HSA)}

A partir da otimização das condições cromatográficas foram determinados os parâmetros cromatográficos. A medida da eficiência da coluna, baseada em pratos teóricos $(\mathrm{N})$, bem como fator de capacidade $(\mathrm{k})$, assimetria de pico a $10 \%$ $\left(\right.$ As $\left._{0,1}\right)$, altura equivalente de pratos teóricos $(\mathrm{H})$, seletividade $(\alpha)$, tempo de retenção $\left(t_{R}\right)$ e da resolução (Rs) foram obtidos a partir do cromatograma ilustrado na Figura 1.

\section{Determinação dos corticosteróides}

A sensibilidade do equipamento em relação aos corticosteróides foi avaliada através da determinação do limite de detecção (LD) e do limite de quantificação (LQ), após a escolha da fase móvel adequada. Tais parâmetros foram determinados utilizando-se concentrações decrescentes de corticosteróides (cortisona, corticosterona, acetato de dexametasona e acetato de hidrocortisona) variando de $0,01 \mu \mathrm{g} \mathrm{mL}^{-1}$ a $5 \mu \mathrm{g} \mathrm{mL}^{-1}$.

A linearidade do detector foi avaliada considerando o Fator de Resposta que representa a razão entre sinal-1 $\left(\mathrm{S}_{1}\right)$ e concentração-1 $\left(\mathrm{C}_{1}\right)$.

\section{Preparação da curva analítica}

As soluções padrão de corticosteróides, contendo concentrações variando de 0,01 a 5,0 $\mu \mathrm{g}$
$\mathrm{mL}^{-1}$ foram utilizadas para a construção da curva analítica. Estas foram preparadas efetuando-se a diluição da solução estoque de corticosteróides preparada previamente. Estas amostras foram injetadas cinco vezes diretamente ao sistema de cromatografia líquida equipado com uma coluna cromatográfica ISRP- $\mathrm{C}_{18}$.

\section{Fortificação das amostras de plasma}

As amostras de plasmas foram diluídas em água destilada $(1: 100 \mathrm{v} / \mathrm{v})$, com o intuito de assegurar que os metabólitos e as proteínas fossem eluídas rapidamente da coluna cromatográfica. A essas amostras de plasmas diluídos foram adicionados dissulfito de sódio $(0,01 \mathrm{~g}$ de dissulfito de sódio para cada $10 \mathrm{~mL}$ água) para evitar a decomposição dos corticosteróides no plasma e também foram adicionadas quantidades conhecidas de corticosteróides. As concentrações obtidas foram 0,1 , $0,2,0,4,0,8$ e $1,6 \mu \mathrm{g} \mathrm{mL} \mathrm{m}^{-1}$ para a curva padrão da fortificação e $0,15,0,30,0,60,1,20$ e $1,60 \mu \mathrm{g} \mathrm{mL}^{-1}$ para a determinação da fortificação em amostras de plasma.

Utilizaram-se seis amostras de plasma de rato que foram injetadas diretamente no sistema CLAE equipado com uma coluna cromatográfica ISRP-C $_{18}$.

\section{Avaliação da recuperação de corticosteróides nas amostras de plasma}

Para avaliar o efeito da matriz biológica na calibração do método, amostras de plasma de rato foram fortificadas com as concentrações de $0,15,0,30,0,60,1,20$ e $1,60 \mu \mathrm{g} \mathrm{mL} \mathrm{mL}^{-1}$ de corticosteróides. Os experimentos de recuperação foram avaliados injetando-se cinco replicatas para cada concentração das amostras sangüíneas.

Foram realizadas análises em branco (matriz isenta de fármacos) para cada amostra plasmática. Verificou-se também o efeito do dissulfito de sódio nas amostras plasmáticas.

\section{Estresse agudo induzido por natação}


Os animais foram submetidos a uma única sessão de natação com duração de 5, 15, 30 ou 50 minutos. A natação foi efetuada em uma cuba de vidro $(45 \mathrm{~cm} \times 35 \mathrm{~cm} \times 33 \mathrm{~cm})$ contendo água à temperatura de $24{ }^{\circ} \mathrm{C}$ [27]. Para garantir que os animais nadassem durante todo o período estipulado, cada animal teve em sua cauda um anel metálico pesando cerca de $2 \%$ do seu peso corporal. Imediatamente após a sessão de estresse, os animais foram decapitados em guilhotina.

\section{Coleta e estocagem de sangue}

As amostras sangüíneas (5-6 mL) foram obtidas do tronco encefálico logo após a decapitação dos ratos em frascos heparinizados, sendo centrifugadas a 2.000 r.p.m., durante $20^{\prime}$ a $2^{\circ} \mathrm{C}$, o plasma obtido foi estocado à $-20^{\circ} \mathrm{C}$. Os frascos contendo plasma foram acondicionados em um container (recipiente isotérmico com $\mathrm{t}^{\mathrm{o}}$ estável) para transportar do Departamento de Farmacologia do Instituto de Biociências da Unesp de Botucatu para o Departamento de Química da Faculdade de Ciências da Unesp de Bauru.

\section{RESULTADOS E DISCUSSÃO}

A fase móvel mais adequada para a separação de corticosteróides foi composta por solução tampão fosfato $\mathrm{pH}$ 4,0 e acetonitrila $(65: 35 \mathrm{v} / \mathrm{v})$. De acordo com dados da literatura, a fase móvel composta por acetonitrila e água é muito comum para a determinação de corticosteróides em fluidos biológicos $[6,9,19]$, além de ser usada para controlar a seletividade e a separação de amostras no modo reverso de eluição [20].

$\mathrm{Na}$ tentativa de se buscar, a melhor resolução para os corticosteróides foram realizados estudos quanto às proporções da fase móvel, assim empregou-se as seguintes: tampão fosfato $\mathrm{pH} 4,0$ : acetonitrila: 60: $40(\mathrm{v} / \mathrm{v})$ e $65: 35(\mathrm{v} / \mathrm{v})$. Para cada proporção das fases móveis foram efetuados os cálculos dos parâmetros cromatográficos e assim foi possível optar pela proporção 65: $35(\mathrm{v} / \mathrm{v})$, na qual apresentou uma excelente eficiência na sepa- ração dos corticosteróides e tempo de análise de 20 minutos, no máximo Figura 1.

Vale ressaltar que a amostra de plasma foi diluída 1: 100, para que se pudesse assegurar que os metabólitos e pequenas concentrações de proteínas fossem eluídos rapidamente da coluna cromatográfica.

A resolução mede a qualidade da separação, que pode ser melhorada com a definição da vazão através da coluna no sistema cromatográfico. Desta maneira, a vazão foi avaliada em um

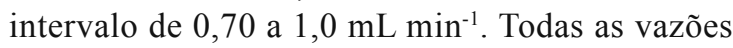
apresentaram uma boa resolução, entretanto a título de tempo de análise e se tratando de fluido biológico, na qual a saída do plasma é em torno de dois minutos, optou-se pela vazão de $0,85 \mathrm{~mL}$ $\min ^{-1}$. No decorrer da fase experimental observase que houve um pequeno aumento no tempo de retenção dos analitos (corticosteróides), fato que pode estar relacionado ao acúmulo de amostras plasmáticas injetados na coluna cromatográfica.

O desempenho da coluna cromatográfica Luna-Phenomenex ISRP-C ${ }_{18}-5 \mu \mathrm{m}$ (100 mm x 4,6 mm DI) foi avaliada em função dos parâmetros apresentados na Tabela 1 (tempos de retenção $\left(\mathrm{t}_{\mathrm{R}}\right)$, número de pratos teóricos $(\mathrm{N})$, altura equivalente a um prato teórico $(H)$, resolução $\left(R_{s}\right)$, seletividade $(\alpha)$, fator de capacidade $(\mathrm{k})$ e assimetria de pico (As) dos diferentes corticosteróides), calculados a partir da Figura 1. De acordo com os resultados obtidos pode-se constatar bom desempenho cromatográfico, uma vez que a imobilização da HSA sobre a superfície externa das partículas esféricas da fase estacionária $\mathrm{C}_{18}$ auxiliou na obtenção de picos bem resolvidos e separação dos corticosteróides do plasma. 
Tabela 1. Parâmetros cromatográficos calculados para os corticosteróides, a partir do cromatograma ilustrado na Figura 1.

\begin{tabular}{cccccccc}
\hline ANALITO & $\mathbf{t}_{\mathbf{R}}(\mathbf{m i n})$ & $\mathbf{N}$ & $\mathbf{H}(\mathbf{m m})$ & $\mathbf{R}_{\mathbf{s}}$ & $\alpha$ & $\mathbf{K}$ & As \\
\hline Cortisona & 3,18 & 1401 & 0,1 & - & 1,8 & 2,8 & 1 \\
Corticosterona & 5,04 & 880 & 0,1 & 4,1 & 1,5 & 5,1 & 1 \\
Ac. Hidrocortisona & 7,29 & 1178 & 0,1 & 5 & 1,8 & 7,8 & 1 \\
Ac. Dexametasona & 12,48 & 863 & 0,1 & 9,4 & - & 14 & 1 \\
\hline
\end{tabular}

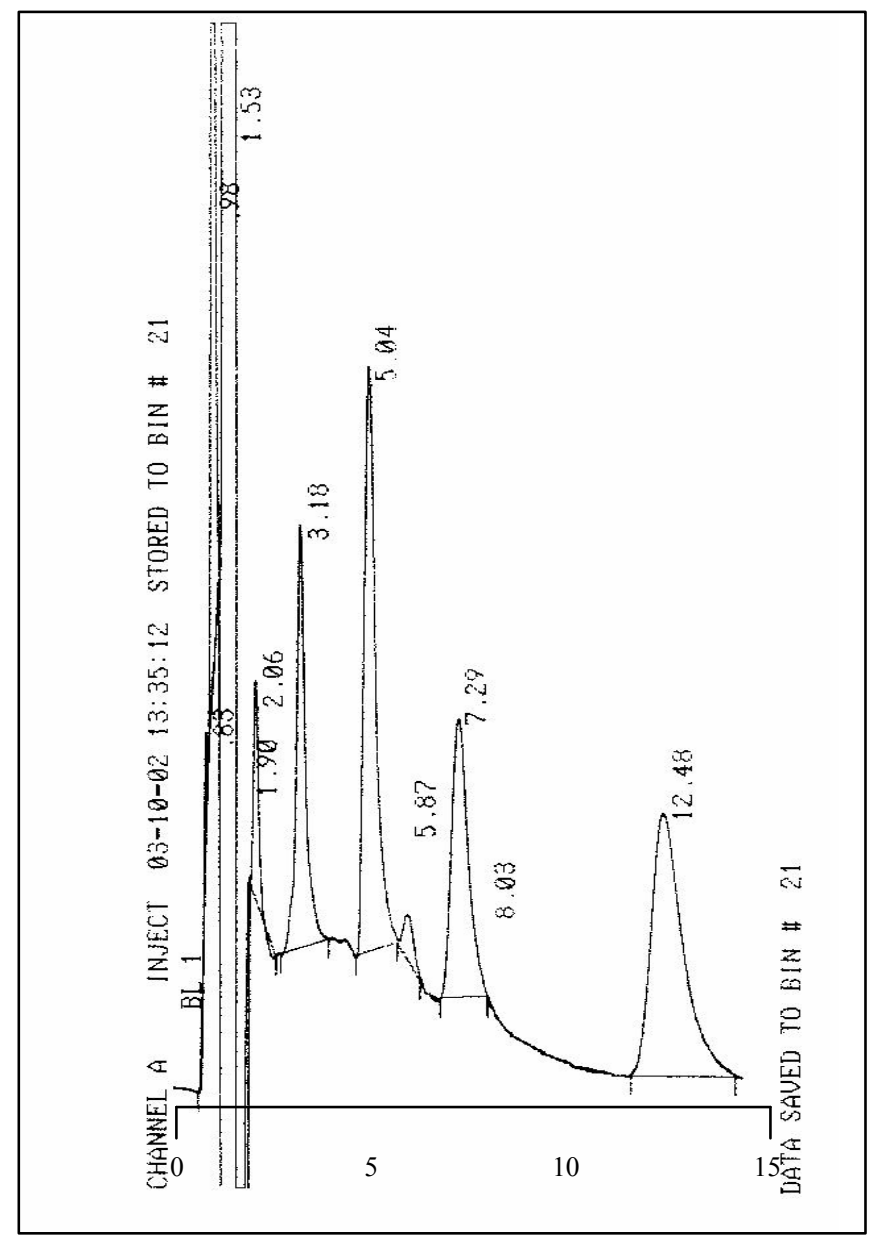

Figura 1. Cromatograma obtido após injeção de $100 \mu \mathrm{L}$ de plasma diluído 1: $100 \mathrm{v} / \mathrm{v}$ fortificado com uma solução de $0,60 \mu \mathrm{g} \mathrm{mL}^{-1}$ de corticosteróides (1-cortisona, 2-corticosterona, 3-acetato de hidrocortisona e 4-acetato de dexametasona) empregando fase móvel composta por tampão fosfato $\mathrm{pH}$ 4,0: acetonitrila (65: $35 \mathrm{v} / \mathrm{v}$ ), vazão de $0,85 \mathrm{~mL} \mathrm{~min}^{-1}$ e detecção a $240 \mathrm{~nm}$. 
Analisando as equações das retas obtidas (Figura 2), observou-se que o modelo linear para a determinação de corticosteróides por CLAE com detector ultravioleta é adequado, já que os coeficientes de correlação foram 0,9991 para a cortisona, 0,9996 para a corticosterona e o acetato de dexametasona e 0,9993 para o acetato de hidrocortisona, como pode ser observado na Tabela 2. Estes valores expressam a relação entre e as áreas dos picos cromatográficos nos intervalos utilizados (y) e as concentrações dos corticosteróides (x). A relação de x e y na curva é expressa como $\mathrm{r}^{2}$ (coeficiente de correlação), onde os valores ideais esperados são 1 e -1 , ou seja, quanto mais próximo da unidade, maior a probabilidade de existir uma relação linear bem definida [21, 22]. Entretanto, a linearidade é melhor avaliada pelo coeficiente de variação (CV\%) do fator de resposta (área/concentração), sendo que o CV deverá ser inferior a $5 \%$.
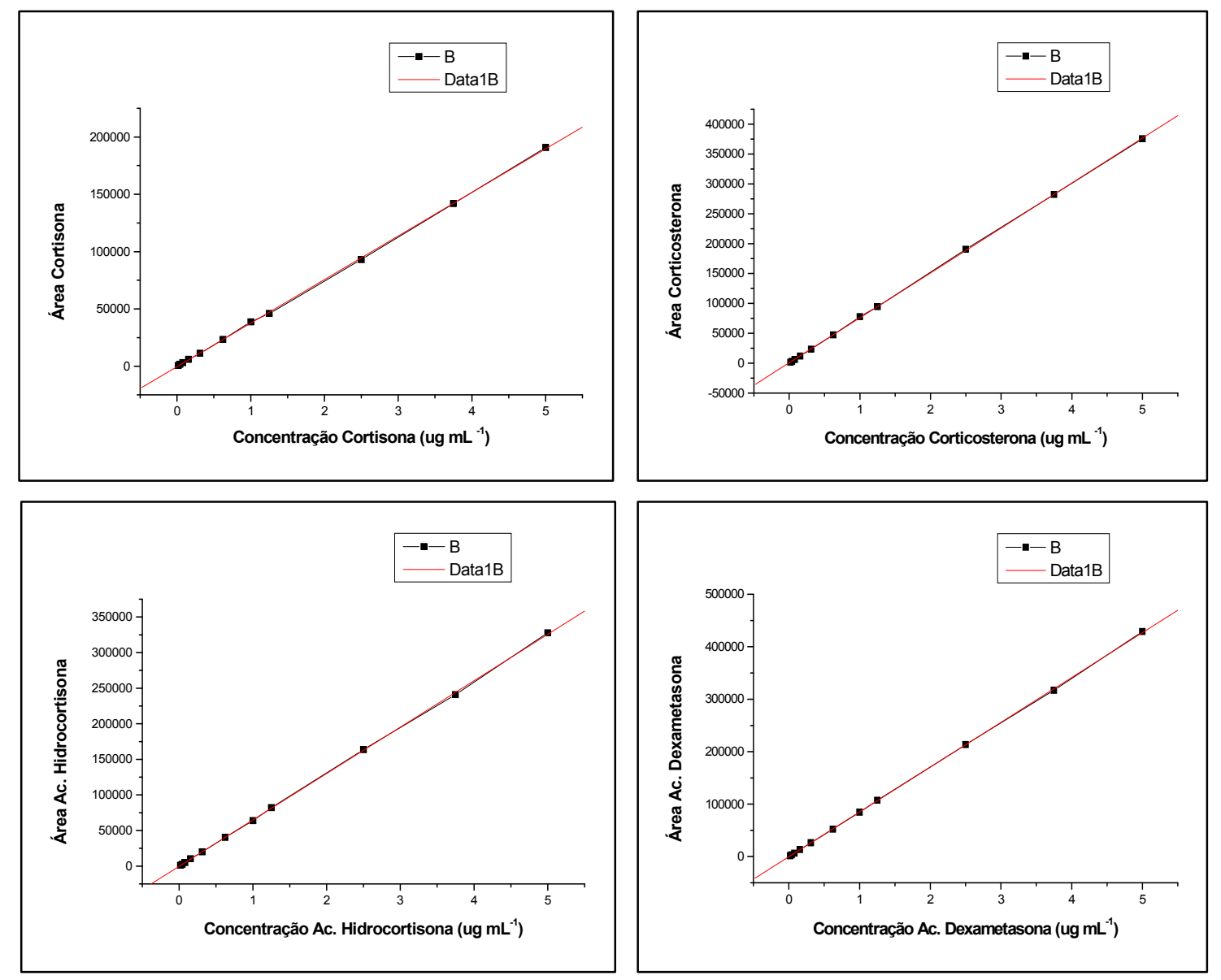

Figura 2. Curvas analíticas de acetato de cortisona, corticosterona, acetatos de dexametasona e hidrocortisona os parâmetros obtidos após a regressão linear. Valores obtidos após a injeção de $100 \mu \mathrm{L}$ da solução padrão de cada costicosteróide no sistema cromatográfico no intervalo de concentração de 0,02 a 5,00 $\mu \mathrm{g}$ $\mathrm{mL}^{-1}$. * média de 5 replicatas para cada concentração. 
Tabela 2. Equação da curva analítica dos corticosteróides.

\begin{tabular}{|c|c|c|c|}
\hline ANALITO & $\begin{array}{c}\text { Equação de regressão } \\
\text { linear }\end{array}$ & Coeficiente correlação & Coeficiente variação \\
\hline Cortisona & $y=-314,61+37986,33 \cdot x$ & 0,9991 & 2,5 \\
\hline Corticosterona & $y=589,67+75210,15 \cdot x$ & 0,9996 & 1,1 \\
\hline Ac. Hidrocortisona & $y=-222,30+65160,41 . x$ & 0,9993 & 1,6 \\
\hline Ac. Dexametasona & $y=-368,16+85426,52 \cdot x$ & 0,9996 & 1,4 \\
\hline
\end{tabular}

$\mathrm{x}$ é expresso em $\mu \mathrm{g} \mathrm{mL}^{-1} \mathrm{e}$ y é expresso em unidade de área.

Diante dos resultados obtidos na Figura 2 e Tabela 2, pode observar que para todos os corticosteróides o coeficiente de variação esteve abaixo de $5 \%$ e o coeficiente de correlação (r) superiores a 0,999 , indicando que há linearidade entre as concentrações (x) e as áreas (y) dos picos no intervalo utilizado $[23,24]$.

Com a aplicação da regressão linear concluiu-se que se pode trabalhar com concentrações de corticosteróides $(0,02,0,04,0,08,0,16,0,31$, $0,63,1,0,1,25,2,50,3,75$, e $\left.5,0 \mu \mathrm{g} \mathrm{mL}^{-1}\right)$ nos extremos da reta obtida e dentro da mesma com valores de coeficiente de variação aceitáveis.

O procedimento de extração dos corticosteróides foi realizado utilizando o plasma. $\mathrm{Na}$ diluição das amostras de plasmas em água destilada (1:100) foram adicionados dissulfito de sódio $(0,01 \mathrm{~g}$ de dissulfito de sódio para cada $10 \mathrm{~mL}$ água), para evitar a decomposição dos corticosteróides no plasma. O sal dissulfito de sódio atua como agente antioxidante, sendo muito utilizado na indústria alimentícia, como conservante de alimentos processados para prevenir o crescimento de bactérias tóxicas [25]. Sem a adição do dissulfito de sódio junto à diluição do plasma observouse que os corticosteróides eram decompostos. Outros métodos para evitar a decomposição dos corticosteróides foram utilizados como manter as amostras em gelo sob abrigo de luz. Acredita-se que alguma enzima presente no plasma estava decompondo os corticosteróides adicionados. Vale ressaltar que o dissulfito de sódio não afetou a análise dos corticosteróides.

Para a validação do método foi avaliada a seletividade e a recuperação. Para avaliar a seletividade do método proposto foram comparados os cromatogramas das amostras testemunhas e das amostras fortificadas. De acordo com os resultados obtidos nenhum interferente elui no tempo de retenção das substâncias de interesse. O método proposto para este estudo conseguiu atingir 0,02 $\mu \mathrm{g} \mathrm{mL} \mathrm{m}^{-1}$ e $0,04 \mu \mathrm{g} \mathrm{mL} \mathrm{m}^{-1}$ para os limites de detecção e para o de quantificação, respectivamente. Nossos resultados mostram-se melhores que os apresentados por [26], onde o limite de detecção e quantificação foram de $0,1 \mu \mathrm{g} \mathrm{mL}^{-1}$ e $0,2 \mu \mathrm{g} \mathrm{mL}^{-1}$, respectivamente. Vale ressaltar que a metodologia proposta no presente trabalho não necessita do pré-tratamento da amostra, fato que pode ser imprescindível em análises de substâncias bioativas presentes em fluidos biológicos.

Os métodos que empregam raidioimunoensaio para a determinação de corticosteróides em fluidos biológicos chegam a detectar níveis na ordem de $\mathrm{ng} \mathrm{mL}^{-1}$, entretanto não possuem especificidade quando comparados aos métodos cromatográficos $[11,27,28,29,30]$. Deste modo, o método proposto pode ser uma alternativa na determinação de corticosteróides em laboratórios de análises clínicas, além de ser seguro para as pessoas que o manipulam já que não se trabalha com radioatividade. Outra desvantagem do radioimunoensaio é a importação dos kits de reagentes e o número limitado de análises, sendo muitas vezes economicamente inviáveis. Assim, a metodologia proposta possui um custo relativamente menor se comparado ao radioimunoensaio, principalmente se o laboratório possuir um aparelho de cromatografia líquida de alta eficiência.

De acordo com o roteiro de validação de métodos analíticos [31, 32, 33] as recuperações devem estar entre 70 a $110 \%$. Em nossos resultados, a recuperação dos corticosteróides em plasma fortificado de rato ficou entre $90 \%$ e $108 \%$, com CV entre 1,1 e 2,5\%, como pode ser observado nas Tabelas 2 e 3, para os níveis de fortificações de $0,15,0,30,0,60,1,2$ e $1,60 \mu \mathrm{L} \mathrm{mL}^{-1}$. 
Tabela 3. Recuperação R (\%) dos corticosteróides $\left(\mu \mathrm{g} \mathrm{mL}^{-1}\right)$ em plasma de rato fortificado.

\begin{tabular}{ccccccccc}
\hline & \multicolumn{2}{c}{ Cortisona } & \multicolumn{2}{c}{ Corticosterona } & $\begin{array}{c}\text { Acetato de } \\
\text { Hidrocortisona }\end{array}$ & $\begin{array}{c}\text { Acetato de } \\
\text { Dexametasona }\end{array}$ \\
\hline Conc $(\boldsymbol{\mu} \mathbf{g} \mathbf{~ m L - 1 )}$ & $\mathbf{R}(\%)$ & $\mathbf{D P R}$ & $\mathbf{R}(\%)$ & $\mathbf{D P R}$ & $\mathbf{R}(\%)$ & $\mathbf{D P R}$ & $\mathbf{R}(\%)$ & DPR \\
1,6 & 97 & 11,6 & 94 & 9,5 & 90 & 9,1 & 97 & 12,7 \\
1,2 & 103 & 7,7 & 103 & 4,4 & 98 & 6,6 & 103 & 7,8 \\
0,6 & 97 & 10,8 & 106 & 4,4 & 101 & 5,7 & 106 & 6,3 \\
0,3 & 103 & 4,4 & 108 & 5,7 & 107 & 5,8 & 105 & 7,5 \\
0,15 & 102 & 6,9 & 102 & 8,9 & 105 & 4,0 & 105 & 4,5 \\
\hline
\end{tabular}

Os resultados estão expressos em média, o desvio-padrão relativo é expresso pelo desvio-padrão dividido pela \% de recuperação x 100 . Foram consideradas 5 análises para cada concentração de corticosteróides.

Várias técnicas para a determinação de corticosteróides em fluidos biológicos necessitam do prétratamento da amostra $[6,9,19,26,34,35,36,37,38,39,40,41]$ seja através de métodos de extração em fase sólida ou extração por solventes (diclorometano, acetato de etila, metanol, entre outros). Vale ressaltar que o pré-tratamento da amostra pode induzir a alguns problemas, como: o tempo dispensado no processo, manuseio laborioso, erros de manipulação, contaminação por outros agentes, alto custo, decomposição de alguns compostos, entre outros. Esses fatores são imprescindíveis em análises rotineiras de um grande número de amostras. O presente método analítico desenvolvido dispensa o pré-tratamento da amostra, fato importantíssimo no diagnóstico e no tratamento de doenças endócrinas relacionadas com os corticosteróides, na qual se faz necessário a rapidez na quantificação destes hormônios. Para validar o método utilizou-se a dosagem dos níveis plasmáticos de corticosterona; hormônio secretado em ratos; após estresse agudo induzido por natação, no qual observou-se um aumento significativo em todos os tempos $(5,15,30$ e 50 minutos) quando comparados ao grupo controle, não havendo diferenças significativas entre os tempos confrome a Tabela 4.

Tabela 4. Níveis plasmáticos de corticosterona $\left(\mu \mathrm{g} \mathrm{mL}^{-1}\right)$ do grupo controle e dos submetidos ao estresse agudo induzido por natação por 5', 15', 30' e 50 '.

\begin{tabular}{ccc}
\hline GRUPOS & $\begin{array}{c}\text { Níveis Plasmáticos } \\
\text { de Corticosterona }\end{array}$ & $\begin{array}{c}\text { Níveis Plasmático de } \\
\text { Corticosterona } \\
\text { (Diluição 1:50) }\end{array}$ \\
Controle & $0,4 \pm 0,1$ & $0,008 \pm 0,002$ \\
EN 05, & $5,1 \pm 1,2$ & $0,102 \pm 0,03$ \\
EN 15, & $4,7 \pm 2,7$ & $0,094 \pm 0,05$ \\
EN 30' & $4,9 \pm 2,7$ & $0,098 \pm 0,05$ \\
EN 50, & $4,7 \pm 2,0$ & $0,094 \pm 0,04$ \\
\hline
\end{tabular}

Valores expressos como média \pm erro padrão de 5 experimentos tanto do grupo controle como do grupo submetido ao estresse induzido por natação. $\mathrm{p}<0.05$, ANOVA seguida de Tukey-Kramer. 
O aumento dos níveis de glicocorticóides é considerado um mecanismo endócrino de primeira linha para defender o organismo contra as condições estressantes [42]. O aumento de glicocorticóides está relacionando a rápida mobilização de gorduras de suas reservas celulares, tornando-os disponíveis para o fornecimento de energia e para a síntese de outros compostos, incluindo glicose, necessários para os diferentes tecidos do organismo $[43,44]$.

Assim, os resultados obtidos foram próximos aos encontrados em métodos descritos pela literatura especializada, mostrando-se em concordância com os valores aceitáveis para a validação de nova metodologia em amostras biológicas. padrão, o qual foi empregado com sucesso para a avaliação das amostras reais.

Os resultados obtidos através do estudo de recuperação estão de acordo com valores aceitáveis para a validação de uma nova metodologia analítica em amostras biológicas, assegurando a eficiência e a confiabilidade do método.

$\mathrm{O}$ método analítico proposto para a determinação de corticosteróides em amostras de plasma empregando um sistema isocrático de cromatografia líquida de alta eficiência com um detector UV-visível e a aplicação de uma coluna cromatográfica ISRP-C ${ }_{18}$ possibilitou a injeção direta (on-line) da amostra sem tratamento prévio, constituindo um método simples, rápido, exato e preciso.

\section{AGRADECIMENTOS}

$\mathrm{Na}$ determinação de corticosteróides em plasma de ratos, foi observado o efeito de matriz, na qual a avaliação da recuperação de corticosteróides foi ajustada utilizando o método de adição
Os autores agradecem a CAPES pelo apoio financeiro, para o desenvolvimento deste trabalho.

\begin{abstract}
The present work had as aim the development of simple an analytical method, fast efficient and the determination of corticosteroids (cortisone, corticosterone, acetate of hidrocortisone and acetate of dexametasone) in sanguine samples of rats using an isocratic system of liquid chromatography of high efficiency (CLAE) with ultraviolet detention. The method involved direct injection of sanguine sample in a chromatographic column with internal surface of phase reverse (ISRP-C18), using the composed mobile phase for drain plug phosphate $\mathrm{pH}$ 4,0: acetonitrile $(65: 35 \mathrm{v} / \mathrm{v})$. The detention the analytes was obtained using an ultraviolet detector of changeable wave length (Varian Model 2550) adjusted in $240 \mathrm{~nm}$ and a $4400 \mathrm{in}-$ tegrator SP Chromaject (Varian Associates, Inc., Sunnyvale, CA, USA). The extraction of the analyte resulted in recovery values between $90 \%$ and $108 \%$, and variation coefficient enter $1,1 \%$ and $2.5 \%$. The detention and quantification limits of the method were 0,02 and $0,04 \mu \mathrm{g}$ $\mathrm{mL}^{-1}$, respectively. In conclusion, the analytical method possible the direct injection (on-line) of the sample without previous treatment also presenting some advantages, such as: fast, exactness, precision and specific.
\end{abstract}

Keywords: corticosteroids, sanguine samples, column ISRP- $\mathrm{C}_{18}$, CLAE.

\section{REFERÊNCIAS}

[1] K. SHIMADA, K. MITAMURA, T. HIGASHI. J. Chromatogr. A, 935 (1-2) (2001) 141-172.

[2] W. ARLT, P. M. STEWART. Endocrinol Metab Clin North Am. 34(2) (2005) 293-313, viii.

[3] J. M. CONNELL, E. DAVIES. J. Endocrinol. 186(1) (2005) $1-20$.
[4] V. CIRIMELE, P. KINTZ, V. DUMESTRE, J. P. GOULLE, B. LUDES. For. Sc. Intern. 107 (1-3) (2000) 381-388.

[5] M. FUJITAKA, S. NOMURA, N. SAKURA, K. UEDA, R. MATUURA, C. YUMIBA. Clin. Chimica Acta, 299 (1-2) (2000) 101-108.

[6] M. HAY, P. MORMÈDE. J. Chromatogr. B. 702 (1-2) (1997) 33-39.

[7] H. SHIBASAKI, T. FURUTA, Y. KASUYA. J. Chromatogr. B. 692 (1) (1997) 7-14. 
[8] A. MARWAH, P. MARWAH, H. LARDY. J. Chromatogra. B. 757 (2001) 333-342.

[9] A. SANTOS-MONTES, R. IZQUIERDO-HORNILLOS. J. Chromatogra. B. 724 (1) (1999) 53-63.

[10] R. A. M. VAN DER HOEVEN, A. J. P. HOFTE, M. FRENAY, H. IRTH, U. R. TJADEN, J. VAN DER GREEF, A. RUDOLPHI, K. S. BOOS, G. M. VARGA, L. E. EDHOLM. J. Chroma. A, 762 (1-2) (1997) p. 193-200.

[11] G. CARPENÉ, A. VETTORETTI, F. PEDINI, S. ROCCO, F. MANTERO, G. OPOCHER. J. Chroma. A. 553 (1991) 201-204.

[12] S. C. N. QUEIROZ, C. H. COLLINS, I. C. S. F. JARDIM. Química Nova. 24 (1) (2001) 68-76.

[13] D. T. ROSSI, N. ZHANG. J. Chroma. 885 (2000) 97113.

[14]M. L. MENEZES, G. FÉLIX, A. C. C. O. DEMARCI. Chromatogra. 47 (1/2) (1998) 81-83.

[15] A. POMPON, I. LEFEBVRE, J. L. IMBACH. Biochem. Pharma. 43 (8) (1992) 1769-1775.

[16] A. SANCHEZ, E. A. TOLEDO-PINTO, M. L. MENEZES, O. C. M. PEREIRA. Pharma. Resea. 50 (2004) 481-485.

[17]A. SANCHEZ, M. L. MENEZES, O. C. M. PEREIRA. Salusvita. 20 (2) (2001) 63-71.

[18] M. L. MENEZES, A. SANCHEZ, P. R. MARTINS, M. Z. GARCIA, O. C. M. PEREIRA, A. A. CARDOSO. Salusvita. 18 (2) (1999) 35-42.

[19] R. G. LUMBRERAS, R. I. HORNILHOS. J. Chroma. B. 742 (2000) 47-57.

[20] Q. B. CASS, A. L. G. DEGANI. São Carlos: Edufscar, (2001) 49-55.

[21] F.LEITE, Validação em análise química, Átomo, Campinas, 4 ed., 2002.

[22] R.CAUSON, J. Chromatogr. B, 689 (1) (1997) 175.

[23] B. M. ERIKSSON, M. WIKSTRÖM. J. Chroma. 593 (1992) 185-190.

[24] P. NI, F. GUYON, M. CAUDE, R. RESSET. J. Liq. Chroma. 12 (10) (1989) 1873-1888.

[25] A. G. GOODMAN, L. S. GILMAN. 9th ed. New York: McGraw-Hill, (1996).

[26] R. MEHVAR, R. O. DANN, D. A. HOGANSON. J. Pharma. Biom. Anal. 22 (2000) 1015-1022.

[27] A. SANCHEZ, O. C. M. PEREIRA. Pharmacological Research. 46 (2002) 56-60.

[28] E. L. SAINIO, A. LEHTOLA, P. ROININEN. Steroids. 51 (1988) 609-622.

[29] B. T. HOFREITER, J. P. ALLEN, A. C. MIZERA, C. D. POWERS, A. M. MASI. Steroids. 39 (1983) 547-555.

[30] S. E. CALVANO, R. W. REYNOLDS, L. D. KEITH. Steroids. 36 (1980) 355-363.

[31] UNITED STATES PHARMACOPEIA CONVENTION. Pharmacopeia 24: validation of compendial methods 1225 (1999).

[32] P. CHIAP, P. H. HUBERT, B. BOULANGER, J. CROMMEN. Analytica Chimica Acta. 391 (1999) 227-238.
[33] M. E. SWARTZ, I. R. KRULL. Pharmacology Tecnology. (1998) 12-19.

[34] T. FURUTA, C. MORI, A. SUZUKI, A. Y. SHIBASAKI, Y. KASUYA. Journal of Chromatography B. 801 (2004) 165171.

[35] N. J. SCHOEMAKER, J. WOLFSWINKEL, J. A. MOL, G. VOORHOUT, M. J. L. KIK, J. T. LUMEIJ, A. RIJNBERK. Domestic Animal Endocrinology. 27 (2004) 13-24.

[36] A. A. KARTSOVA, L. I. VELIKANOVA, E. G. PAVLOVA, E. A. BESSONOVA. Journal of Analytical Chemistry. 59, n.10 (2004) 976-982.

[37] S. ABURUZ, J. MILlERSHIP, L. HEANEY, J. MCELNAY. Journal of Chromatography B. 798 (2003) 193201.

[38] B. C. McWHINNEY, G. WARD, P. E. HICKMAN. Clinical Chemical. 24, n. 6 (1996) 979-981.

[39] S. A. DÖPPENSCHMITT, B. SCHEIDEL, F. HARRISON, J. P. SURMANN. Journal of Chromatography B. 682 (1996) 79-88.

[40] W. J. JUSKO, N. A. PYSZCZNSKI, M. S. BUSHWAY, R. D' AMBROSIO, S. M. MIS. Journal of Chromatography B. 658, n. 1, (1994) 47-54.

[41] A. N. KONG, R. L. SLAUGHTER, W. J. JUSKO. Journal of Chromatography B. 432 (1988) 308-314.

[42] E. MÖSTL, R. H. PALME. Domestic Animal Endocrinology. 23 (2002) 67-74.

[43] A. PEREIRA, C. FREITAS, C. MENDONÇA, J. MARÇAL, J. SOUZA, J. P. NORONHA, L. LESSA, L. MELO, R. GONÇALVES, A. SHOLL-FRANCO. Ciências \& Cognição. 1 (2004) 34-53.

[44] H. LÜLLMANN, K. MOHR, A. ZIEGLER, D. BIEGER D. Pocket atlas of pharmacology: 149 color plates by Jürgen Wirth. New York: Verlag (1993) 235-237. 